\title{
Comparison of mobile source emission models using aggregated and disaggregated data
}

\author{
A. Perez \& A. de Barros \\ Transportation Department, University of Calgary, Canada
}

\begin{abstract}
Mobile source emission models are designed to provide a quantification tool of the amount of pollution that is released to the atmosphere from the vehicles within a defined region. The most common models were developed based on aggregated data, such as vehicle miles travelled, fuel consumption and average travelling speed. Recently, new models have been developed. They use a disaggregated analysis approach in order to include the sudden changes in speed and acceleration and the traffic interactions in the calculation. This paper presents a comparison between three different models developed on three different levels of data aggregation and their application on a road stretch. Traffic data (speed, acceleration and flow) is extracted from a micro simulation model and then used to calculate the total emissions during a specific period of time. Emission data was collected using a Portable Vehicular Emission Measuring System in a chassis dynamometer on a second by second basis. The main purpose of this research is to show the main differences in the calculation of emissions and their applicability to different levels of emission inventories.
\end{abstract}

Keywords: mobile sources, emissions, micro simulation, average speed, instantaneous speed, instantaneous acceleration, air pollution.

\section{Introduction}

All modes of transportation that involve the combustion of liquid fossil fuels are a source of pollutants that are directly emitted to the atmosphere, causing a variety of impacts to the environment [1]. The combustion of fossil fuels, under ideal conditions, produces two main products, which are $\mathrm{CO}_{2}$ and $\mathrm{H}_{2} \mathrm{O}$ (vapour), but in the real world these conditions are not easily replicable and incomplete 
combustion is mostly present in the majority of engines. The incomplete combustion adds more products to the combustion process, which are mainly Carbon Monoxide (CO), Volatile Organic Compounds (VOCs), Particulate matter (PM) and Nitrogen Oxides (NOx).

A major concern for the authorities is to have the ability to quantify the emissions produced in their cities and also characterize them according to the type of source. This information is useful as a basic input to develop different type of policies in the local to regional level. There are numerous factors affecting the type and amount of pollutants that are produced by vehicles. The purpose of the emission factors model is to collect all these variables into a mathematical or statistical model that could be applied to get an accurate estimate of the emissions for a specific area.

Different types of emission models have been developed in order to meet the objectives of the emissions inventories. In a local scale the emissions models have to describe the relationship among the emissions, the driver behaviour and the traffic interaction. In a regional context the use of average statistics allow one to get emission values on large regions more easily [2]. These models can be grouped as aggregated and disaggregated models, according to the level of detail that they include in the analysis.

Depending on the type of model that is developed, the data acquisition can be performed using different techniques. Some of them require setting up large infrastructure facilities, such as the dynamometer tests. A smaller scale can be obtained using remote sensing equipment that allows an easier set up, or using on board equipment, which have a portability characteristic helping to ease the data collection process.

The most widely used technique is performed using a series of dynamometer tests for a considerable amount of vehicles. The dynamometer tries to replicate a real-world situation by following a previously selected and designed driving cycle. Another technique is the on road test, where remote sensing systems are used to measure concentrations on a specific point for long periods of time. This technique includes remote sensing equipment set up on the side of the street, which measures the emissions from vehicles passing that specific point, and a device that records the speed of the vehicles and takes a picture of the plates of the vehicles measured. This technique allows access to a greater variety of data, but at the same time it provides limited data per vehicle, and there is no control over the sample [3]. A different technique is the use of on board equipment that collects information about the engine and the emissions on a second by second basis. This type of technology is relatively new, but there are several studies that have been done using this equipment [4].

There are other techniques that have also been used, such as the tunnels technique, where the total concentration is measured inside a tunnel and the number and type of vehicles going into the tunnel is recorded at the same time. All the previous techniques are not exclusive from one to another, but they can be combined depending on the type of model needed or the infrastructure available for the data collection. 
Air pollution is a big concern in Quito, Ecuador due to the high levels of concentration that are present. This was chosen as an example because it would show how a relatively small city is dealing with air pollution and what alternatives they have to develop new plans and policies towards a better quality of life in the city.

\section{Mobile source emission models}

Mobile source emission models have become a tool for governments to deal with air pollution issues and at the same time to develop new policies oriented to have a more efficient vehicular fleet. Regional or aggregated models are the first choice of authorities due to their simple application and also due to the lack of traffic data available. Few cities have developed more advanced and specific models where the emissions can be associated with a spatial context and used to evaluate more detailed impacts on the quality of life in the city.

Aggregated models are mostly used in a regional context to provide a general idea of the emissions produced within a specific region. The use of few aggregated variables in the model is the main characteristic of this type of model. Some of these models relate emissions with the number of trips, and distance travelled, while others relate the total fuel consumption in the region and the total emissions produced for that amount of fuel. This type of model has the disadvantage of being developed for an area and not being able to be geographically distributed within the area. The most widely used models are the MOBILE 5.0 and 6.0, and their variations, from the US Environmental Protection Agency, as well as the EMFAC 2000 [5]. These models are based mainly on the activity and characteristics of the vehicle. The vehicle characteristics, in most cases, are: the type, the age and the average speed of the vehicle, as well as the temperature, altitude, vehicle load, air conditioner usage and operating mode, and the vehicle activity variables are the miles travelled and number of trips, among others. The emissions are developed using a specific driving cycle that tries to represent the real world vehicle behaviour on a laboratory under a controlled environment. One disadvantage of these models is that if the traffic system has a different average speed from the one that the model was developed for, the model uses a group of Speed Correction Factors that are derived from a set of limited driving cycles that do not necessarily represent real world traffic. Another disadvantage is that the FTP driving cycle is twenty years old, and it needs to be updated in order to respond to new driving conditions [6]. MOBILE 6.0 has features that help it to be more accurate, they are the off cycle emissions, higher speeds and accelerations, the inclusion of evaporative diurnal emission factors and the effects of national low-emissions vehicles, among others.

Aggregated models do not have the compatibility to be used to evaluate the environmental impacts of projects focused on modifying traffic behaviour [7]. These types of models are not sensitive to certain vehicles modes of operation, such as idling, cruising, acceleration and deceleration [8]. The variation in average speeds, that is the input variable for these types of models, is not very 
sensitive and does not represent the variation of instantaneous speeds. The second variable that most of these models use the distance driven, and this variable is not going to change unless there are deviation projects where the traffic is diverted to alternative routes.

Air pollution in a local context is influenced by a long list of pollution sources, and in order to have control and monitor this phenomenon it is important to have the resources that help to allocate correctly the sources of pollution [9]. Under a local context, it is necessary to have a tool that allows evaluation of policies and traffic changes from an air pollution point of view, because these impacts are going to have a consequence on the community as well. Disaggregated mobile source emission models are designed to give alternatives for this type of application where the level of detail is higher than the regional applications.

Disaggregated models have as a main characteristic a high sensitivity to changes in instantaneous speed, acceleration and vehicular flow. This type of model is very location specific, because it is influenced by environmental and physical variables that are also specific for each city or region. There are different types of models that have been developed lately, some are developed based on the modes of operation and some of them are related to instantaneous speed and acceleration in general. There are also variations of the models where the authors include more variables such as slope, engine power, and vehicle load. The greater the number of variables that the model has, the higher the sample size is needed.

The inclusion of instantaneous variables, such as speed and acceleration, is necessary in order to be able to represent the changes on a micro-scale context [10]. These small scale scenarios are affected by traffic control and management strategies that are designed to change the modal events on a network, hence instantaneous speed and acceleration are influenced (8). The influence of speed fluctuation on emission levels is relatively important for local emissions inventories [11].

The University of California develop the CMEM model where the emissions are related to the vehicle operation mode. This model was developed with a 300 vehicles sample tested on a dynamometer using the FTP and the US06 driving cycle. The variables included in the model are the speed, acceleration, grade, cold start, engine friction factor among others [12]. Alternatively the University of Virginia developed its own model called VT Micro. Similar to CMEM this was developed using dynamometer tests but the relations were defined between the emissions and the instantaneous speed and acceleration. The final model has a combination of linear, quadratic, cubic and quadratic terms of speed and acceleration. This model reports good measures of fit in relation with fuel consumption and NOx but low values in relation with $\mathrm{HC}$ and $\mathrm{CO}$ [7].

During the last years, the on board data collection technique has been improved in a way that it appeared as an alternative process that allows to collect real world emissions data. This technology is fairly new and its costs are high, and that is the reason why there are not many studies with this kind of data. The Regional Association of Oil and Gas companies in Latin America and the 
Caribbean (ARPEL) have conducted a study measuring instantaneous emissions in relation with speed and acceleration in three different cities: São Paulo, Santiago de Chile and Buenos Aires. This study is oriented to test the different types of fuels and its pollution characteristics [4]. The number of vehicles measured is 25 in each city using a determined driving cycle.

The Environmental Protection Agency of United States has been working on MOVES, a new approach that includes the disaggregated approach. It has been developed but it has not been promoted as MOBILE. MOVES has been developed to be able to be applied under a regional scale as well as local scale [13].

The benefits of measuring the instantaneous speed and acceleration of a vehicle in operation are that the driving behaviour can be defined on a more realistic way. Sudden changes of acceleration to deceleration as well as stop and go traffic can be measured and included in the model. One advantage of this technique is the ability to include different engine parameters as well as emission values in the model. This technique is a more realistic approach to normal traffic congestion but as disadvantage it has a great data variation than the other techniques. If the measurements are set up using a GPS system it could provide information to analyse the level of influence of slopes and spatial configuration on the emissions.

\section{Experimental}

This research was conducted in the city of Quito in Ecuador. Emission data was collected as well as traffic data from the city. The emissions data collection campaign was part of the emissions inventory program that the Air Quality Improvement Corporation (CORPAIRE) conducts each year as part of the Air Quality Management Network of the City. Traffic data was provided by the Department of planning of the municipality.

\subsection{Emissions data}

The data collection campaign was conducted using a chassis dynamometer located in the southern area of Quito. A total of 23 vehicles were measured using the IM240 driving cycle. Each vehicle was measured three times in order to have a more reliable measurement. The emissions were registered using a Portable Vehicular Emission Measuring System from Clean Air Technologies. This system measures the combustion gases and particulate matter from the tail pipes of the vehicles on a second by second basis. It uses different types of detection methods depending on the type of pollutant. For $\mathrm{HC}, \mathrm{CO}$ and $\mathrm{CO}_{2}$ it uses a non dispersive infrared camera; for NOx it uses an electrochemical cell; and for particulate matter, it uses a light dispersion method with different opacity levels. The total gas flux is measured knowing the engine and fuel properties.

The data had to be screened before analysed looking for records where the equipment could not perform a reading or the values were corrupted. A total of 18813 records were valid and were input into a database to ease their management. 


\subsection{Traffic data}

Traffic data was provided by the municipality of Quito. Traffic counts during peak hours on the four legs of an interchange that connects the northern part of the city with the southern part of the city. Road alignments were drawn using cartographic maps on a 1:5000 scale and Google Earth using digitalization processes. This data was input into SIAS PARAMICS to develop a microsimulation of the vehicular traffic in this area.

A large amount of detectors were inserted along the roads. These detectors register instantaneous speed and acceleration of each vehicle that crosses that point. Distances between detectors were very short in order to emulate a second by second traffic data extraction.

Table 1: $\quad$ List of vehicles tested on the chassis dynamometer.

\begin{tabular}{|l|l|l|l|l|l|l|l|}
\hline & Maker & Year & Tests & & Maker & Year & Tests \\
\hline 1 & Hyundai & 2009 & 3 & 14 & Volkswagen & 2001 & 3 \\
\hline 2 & Hyundai & 2005 & 4 & 15 & Chevrolet & 2002 & 3 \\
\hline 3 & Hyundai & 2002 & 3 & 16 & Chevrolet & 2007 & 3 \\
\hline 4 & Chevrolet & 2002 & 3 & 17 & Chevrolet & 2008 & 5 \\
\hline 5 & Chevrolet & 2005 & 3 & 18 & Chevrolet & 2004 & 1 \\
\hline 6 & Chevrolet & 2008 & 3 & 19 & Chevrolet & 2006 & 3 \\
\hline 7 & Chevrolet & 2006 & 3 & 20 & Kia & 2005 & 3 \\
\hline 8 & Chevrolet & 2007 & 3 & 21 & Chevrolet & 2004 & 3 \\
\hline 9 & Chevrolet & 2007 & 3 & 22 & Chevrolet & 2006 & 3 \\
\hline 10 & Hyundai & 2006 & 3 & 23 & Chevrolet & 2005 & 3 \\
\hline 11 & Hyundai & 2005 & 3 & 24 & Chevrolet & 2005 & 3 \\
\hline 12 & Daewoo & 2001 & 3 & 25 & Chevrolet & 2007 & 3 \\
\hline 13 & Chevrolet & 2007 & 3 & 26 & Volkswagen & 2003 & 3 \\
\hline
\end{tabular}

\section{Results}

An aggregated model was developed using average values of emissions. The data was divided into groups in order to have average values of emissions for speed ranges from $10 \mathrm{~km} / \mathrm{h}$ to $100 \mathrm{~km} / \mathrm{h}$. In this model the variables included were only speed and emissions, and the final units of the emissions were $\mathrm{g} / \mathrm{s}$. The formula applied to calculate the emissions is shown in Eq. 1:

$$
E_{\mathrm{CO} 2}=E F[g / s] \times A v S[m / s] \times d t[m] \times f
$$

where:

$\mathrm{EF}=$ Emission Factor in grams per second

AvS $=$ Average Speed

$\mathrm{Dt}=$ Distance travelled

$\mathrm{f}=$ flow 
A second model was developed using a linear regression approach. The "second by second" data was input into the regression process, some transformations were applied to the variables in order to have a greater set of independent variables in the model. In previous studies $[8,10]$ a combination of speed and acceleration raised to different power showed to be a good approach for this type of regressions. Speed and acceleration were raised to the square and cubic power and combined with each other. In the case of Carbon Dioxide the dependent variable was not transformed, but in the rest of the cases the emissions were transformed using natural logarithm in order to have a better measure of fit of the equation. Table 2 shows the results of those regressions.

Table 2: $\quad$ Regression coefficients for the disaggregated model.

\begin{tabular}{|c|c|c|c|c|c|c|c|}
\hline \multicolumn{2}{|c|}{$\mathrm{CO} 2$} & \multicolumn{2}{|c|}{$\mathrm{Ln} \mathrm{CO}$} & \multicolumn{2}{|c|}{ Ln HC } & \multicolumn{2}{|c|}{ Ln NOx } \\
\hline $\mathrm{S}$ & 0.0458 & $\mathrm{~S}$ & 0.2638 & $\mathrm{~S}$ & 0.1491 & $\mathrm{~S}$ & 0.1932 \\
\hline $\mathrm{a}$ & 0.8523 & $\mathrm{a}$ & 2.8945 & $\mathrm{a}$ & 2.0669 & $\mathrm{a}$ & 0.3123 \\
\hline s2 & 0.0273 & $\mathrm{~s} 2$ & -0.0193 & s2 & -0.0041 & s3 & -0.0001 \\
\hline s3 & -0.0008 & s3 & 0.0004 & $\mathrm{a} 2$ & -0.2152 & a2 & 0.2323 \\
\hline $\mathrm{a} 2$ & 0.3586 & $\mathrm{a} 2$ & -0.0832 & a3 & -0.7813 & as & 0.0219 \\
\hline a3 & 0.1623 & a3 & -0.9110 & as & -0.8225 & $\mathrm{a} 3 \mathrm{~s}$ & 0.0301 \\
\hline as & -0.1181 & as & -1.2024 & a2s & 0.0995 & a2s2 & -0.0013 \\
\hline as2 & 0.0044 & $\mathrm{a} 3 \mathrm{~s}$ & 0.5353 & $\mathrm{a} 3 \mathrm{~s}$ & 0.3996 & a3s2 & -0.0020 \\
\hline $\mathrm{a} 2 \mathrm{~s} 2$ & -0.0047 & as2 & 0.1258 & as2 & 0.0821 & age & 0.3072 \\
\hline $\mathrm{a} 3 \mathrm{~s} 2$ & 0.0092 & as3 & -0.0033 & as3 & -0.0021 & cons & -7.9834 \\
\hline $\mathrm{a} 3 \mathrm{~s} 3$ & -0.0004 & a2s3 & 0.0001 & a2s2 & -0.0113 & & \\
\hline age & -0.0274 & a3s2 & -0.0561 & a2s3 & 0.0004 & & \\
\hline cons & 2.7613 & a3s3 & 0.0015 & a3s 2 & -0.0389 & & \\
\hline & & age & 0.2493 & a3s3 & 0.0010 & & \\
\hline & & cons & -5.4040 & age & 0.1344 & & \\
\hline & & & & _cons & -7.0195 & & \\
\hline
\end{tabular}

A third model was developed by separating the data according to the acceleration mode as it was suggested by previous literature [5, 10]. Three operation modes were selected acceleration, deceleration and idle. Following the same concept of the second model, a linear regression was performed with each subset. A set of three equations was developed for each pollutant. The independent variable in this approach was the speed and a combination of acceleration and speed.

The traffic micro-simulation was used to extract instantaneous speed and acceleration data. A large number of detectors were inserted in the model in order to simulate a second by second information extraction. The simulation was done in a corridor without traffic lights in order to show in a more appropriate way how the speed and acceleration profiles influence the emissions. A total of 
261 detectors were located along the interchange. The micro-simulation output had to be processed in order to be compatible with the emissions models.

For the aggregated approach, the network was divided in sections where the flow was constant, and the average speed was calculated using the detectors located in the correspondent section. Length of the section was calculated from the alignment drawing. For the disaggregated approach each record was input into the regression model and a total emission per detector was calculated. The total emissions of the system were calculated by summing up the totals for each detector. In the case of the modal approach, the data was classified by value of acceleration (acceleration, deceleration, Idle) and the equations were applied to each record. Table 3 contains the total emissions of the network in tons.

Table 3: $\quad$ Total emissions from the network.

\begin{tabular}{|l|l|l|l|l|}
\hline & NOx & HC & CO & CO2 \\
\hline Aggregated & 11.51546 & 5.164909 & 82.5069 & 8890.331 \\
\hline Disaggregated & 1.444284 & 5.7345 & 75.72485 & 914.6014 \\
\hline Modal & 1.354915 & 4.692205 & 39.63877 & 900.8564 \\
\hline
\end{tabular}

\section{Analysis}

Table 3 shows the values of emissions calculated for the simulation. Carbon Dioxide emissions are significantly higher when the aggregated model was applied. This could be explained due to the variability of the speed profile. This model was developed using average emission values grouping the data each 10 $\mathrm{km} / \mathrm{h}$. More elaborated models count with a correction factor for different speeds, this assumption can explain the overestimation of $\mathrm{CO}_{2}$ because all the averages speed were approximated to the higher multiple of 10. The lack of more appropriate data was a barrier in order to have a better aggregated mode. Hydrocarbons (HC) and Carbon Monoxide have similar values compared to the disaggregated and modal approach. Nitrogen Oxides (NOx) have also a high difference, this could be explained by the lack of more explanatory variables in the regression model and the low goodness of fit (R-square $=0.34$ ). The formation of NOx is produced by the surplus of air in the combustion at high temperatures. A better NOx model can be calculated having variables such as air-fuel ratio included in the model.

If total emissions are compared between the disaggregated model and the modal approach, they show similar values with small differences. The level of detail of the modal approach seems to give high values for all the pollutants. Fig. 1 shows an example of the emission patterns on a section of the road, the three models are shown.

Fig 1 shows the variability on the emissions calculation. The aggregated model that assumes an average speed along the road and calculates a unique value, the disaggregated model has a higher variation in relation to variation of the speed. When the speed increases in Loop 063 and forward the emissions start decreasing significantly. The modal approach shows a more stable model where 
the variations are not very significant, the increase on speed also causes a decrease on emissions but as it also depends on the acceleration mode this variation is not as big as in the deceleration mode. After Loop 057 there is an increment of flow and this causes the increment on emissions. If we compare the speed profiles are very similar before and after this point but the emissions do change along the road.

Emission patterns are similar between the disaggregated and the modal approach but the modal approach always has higher values than the disaggregated approach. Previous studies (13) showed that a classification by operation modes give a better accuracy to the model. New models such as MOVES have been developed using this technique in order to provide more accurate emissions values. This approach allows including the interaction between acceleration and speed without having to have instantaneous acceleration data.

The application of traffic management (TM) techniques has to be oriented to reduce traffic but they also have to be analysed from an environmental point of view because sometimes it could help to reduce traffic in one place but it can cause to move the pollution from one point to another point where the pedestrian are in more direct contact. In fig. 1 we can see that for example a good TM technique could be to reduce traffic after Loop 057 to levels similar at those that are present after Loop 064.

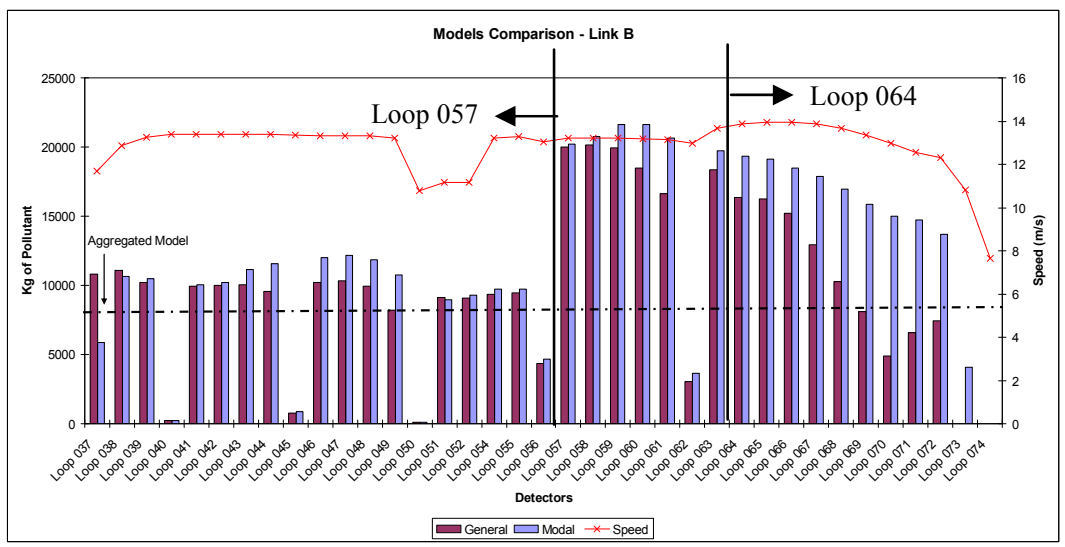

Figure 1: $\quad$ Emissions distribution on link B.

\section{Conclusions and recommendations}

This paper shows the differences that different models can have in the calculation of emissions from mobile sources. The key factor, to decide which approach is more appropriate, is the final use of the emission inventory, and its application. Aggregated models are more suitable for regional inventories that provide a general idea on how much pollutants are emitted to the atmosphere in a 
specific region. A disadvantage of this type of models is that are not suitable to be combined with air quality models because the emissions do not have a spatial context in which to be analysed. Alternative units are mass of pollutant per litre of gasoline consumed, and they are used in combination with the records of fuel sales in the region. The main barrier to apply this alternative is that those records are not always available and accurate. The benefits of these aggregated models are that are less expensive compared to disaggregated models, and they can provide a good general snapshot of the level of contribution of pollution from the mobile sources.

Pollutant emissions caused by mobile sources contribute to worse air pollution problems in a city or a region. Some cities have to deal with high levels of air pollution and they do not always have a tool that allows them to evaluate the alternatives they have in traffic management. Disaggregated models have as main benefit to give a spatial context to the emissions. This allows to see the spatial distribution of the emissions and to locate hot spots where high levels of emission are present in a micro scale level. The application of Intelligent Transportation Systems, for example, is mainly oriented to change the driving patterns in a specific corridor within a city or region and aggregated models would not represent the impacts that those projects can cause on emissions and air pollution. Disaggregated models provide the authorities a tool to evaluate micro-scale projects and visually see how the changes impact the population.

\section{References}

[1] Colvile RN, Hutchinson EJ, Mindell JS, Warren RF. The transport sector as a source of air pollution. Atmospheric Environment 2001 3; 35(9):15371565.

[2] Sturm P, Pucher K, Sudy C, Almbauer R. Determination of traffic emissions - intercomparison of different calculation methods. The Science of the Total Environment 1996; 189:187-196.

[3] Chan TL, Ning Z, Leung CW, Cheung CS, Hung WT, Dong G. On-road remote sensing of petrol vehicle emissions measurement and emission factors estimation in Hong Kong. Atmospheric Environment 2004 5; 38(14):2055-2066.

[4] Rideout G, Gourley D, Walker J. Measurement of in service Vehicle Emissions in Sao Paulo, Santiago and Buenos Aires. August 2005; 252005.

[5] Noland RB, Ochieng WY, Quddus MA, North RJ, Polak JW. The vehicle emissions and performance monitoring system: Analysis of tailpipe emissions and vehicle performance. Transp. Plann. Technol. 2004; 27(6):431-447.

[6] Rakha H, Ahn K, Trani A. Comparison of MOBILE5a, MOBILE6, VTMICRO, and CMEM models for estimating hot-stabilized light-duty gasoline vehicle emissions. Canadian Journal of Civil Engineering 2003; 30(6):1010-1021. 
[7] Ahn K, Rakha H, Trani A, Aerde MV. Estimating Vehicle Fuel Consumption and Emissions based on Instantaneous Speed and Acceleration Levels. J. Transp. Eng. 2002; 128(2):182-190.

[8] Yu L. Remote Vehicle Exhaust Emission Sensing for Traffic Simulation and Optimization Models. Transportation Research Part D: Transport and Environment 1998 9; 3(5):337-347.

[9] Pokharel SS, Bishop GA, Stedman DH. An on-road motor vehicle emissions inventory for Denver: An efficient alternative to modelling. Atmos. Environ. 2002; 36(33):5177-5184.

[10] Cappiello A, Chabini I, Nam EK, Lue A, Abou Zeid M. A statistical model of vehicle emissions and fuel consumption. Intelligent Transportation Systems 2002:801-809.

[11] de Haan P, Keller M. Emission factors for passenger cars: application of instantaneous emission modelling. Atmospheric Environment 2000; 34(27):4629-4638.

[12] Barth M, Norbeck J. Transport Modelling for the Environment: Final Report. February, 1996; UCB-ITS-PRR-96-6.

[13] Koupal J, Cumbertworth M, Michaels H, Beardsley M, Brzezinski D. Draft Design and Implementation Plan for EPA's Multi-Scale Motor Vehicle Equipment Emission System (MOVES). 2002. 